\title{
Factors Contributing to Increased Patient Wait Times in Selected Wound Care Patients: A Retrospective Study
}

\author{
Joan Aina $^{1} \&$ Jochebed Ade-Oshifogun ${ }^{2}$ \\ ${ }^{1}$ Adjunct Assistant Professor, Department of Nursing, Andrews University, Berrien springs, Michigan, USA \\ ${ }^{2}$ Associate Professor and Chair, Department of Nursing, Andrews University, Berrien Springs, Michigan, USA \\ Correspondence: Samuel P. Abraham, Assistant Professor of Nursing, Bethel College, Indiana, USA.
}

Received: April 26, 2018

doi:10.20849/ijsn.v3i2.383

\begin{abstract}
Background: 'Waiting' can be frustrating for anyone especially when it comes to healthcare. The Institute of Medicine advocates changes to improve the quality of the health care delivery system in the United States.

Purpose: The purpose of this retrospective study was to determine the factors contributing to increased patient wait times in selected wound care patients. The question guiding this project is-in selected wound care patients who received treatment between September1-December 31, 2013; are factors contributing to prolonged wait times related to treatment-related diagnosis, providers, and clinicians?

Method: After institutional review board approval, retrospective charts review was conducted. 300 charts were randomly selected from the electronic health record (EHR) database at a local hospital wound care clinic. 120 charts met the inclusive criteria and were analyzed using ANOVA and SPSS version 22. The Deming cycle for quality improvement was adopted as the framework for practice review and changes.
\end{abstract}

Result: Among all the factors examined, Treatment diagnosis accounted for $4 \%$ of the variance $(p=0.416)$; Providers $1 \%(p=0.208)$; and Clinicians $8 \%(p=0.195)$. Though clinicians had the highest variance, it was not a significant factor for patient wait times. The Deming cycle helps to prioritize and improve communication by creating a chart for effective patient flow through the clinic to reduce wait time.

Conclusion: Correcting and improving wait times has the potential for increasing timely access and patient satisfaction. Clinicians and providers are not significant factors contributing to wait times. Wait time should be given priority and be regularly reviewed as part of the quality improvement plan within any organization.

Keywords: wound care, wait times; diabetic ulcer, pressure ulcer, venous ulcer, Continuous Quality Improvement (CQI)

\section{Introduction}

Although it can be frustrating, waiting is not a new concept, and just about everyone seeking health care services must experience some wait time. Increased wait times reduce efficiency and can be unbearable; however, those wait times are sometimes necessary for thorough evaluation and to avoid errors. Wait times continue to be a quality improvement issue in many health care settings that is, multifactorial in nature and therefore an aspect of health care that is expected by patients, providers and staff to be at a level acceptable to most patients (Bleustein et al., 2014; McHugh, Van Dyke, McClelland, \& Moss, 2011; Oredsson et al., 2011).

Prolonged wait times have an impact on patients' satisfaction, care, and healing. There is continuous emphasis placed on timely access to care, as evidenced by the Patient Protective Affordable Health Care Act of 2010 (United States of America). The increase in wait times was identified by staff at a community hospital wound care center in 2013 as a quality issue, and a continuous quality improvement (CQI) framework was adopted to improve the perceived increase in wait times (Dinesh, Singh, Nair, \& Renya, 2013; Vasquez, Campbell, Haman, George, \& Sprabery, 2009). The CQI is a basic model for strategic improvement that is focused on customer satisfaction using the Plan-Do-Check-Act Cycle (PDCA; The W. Edward Deming Institute, 2014). A retrospective approach was adopted to identify factors contributing to increased wait times at the wound care center to find ways for clinical improvement.

The purpose of this retrospective study was to determine the factors contributing to increased patient wait times in 
selected wound care patients. The questions guiding this study are:

1) What factors contribute to prolonged wait times in selected wound care patients?

2) Do treatment-related diagnosis, providers and clinicians factors contribute to prolonged wait times?

3) How can we improve wait times with this population?

\section{Literature Review}

\subsection{Patient Satisfaction and Patient Condition}

Researchers have examined wait times in terms of patients' satisfaction with providers and health care institutions, patients' perceptions of wait times, patients' conditions, and tools used to improve wait times (Bleustein et al., 2014; Chen, Chang, Shen, Lin, \& Chen, 2015; Fournier, Heale, \& Rietze, 2012; Murphy \& Evans, 2012). Evidence from literature demonstrates that because of long wait times, patients may leave without being seen by a provider, indicating a need to reduce the wait for patients in many health care settings. Many other studies have examined how satisfied, or dissatisfied patients were when it came to health-care-related wait times (Esbenshade, 2015; Michael, Schaffer, Egan, Little, \& Pritchard, 2013). The general conclusion of the authors is that, longer wait times are strongly associated with lower patient satisfaction. Other studies examining the association between wait times and patient satisfaction and the availability of scheduling personnel on the telephone as one factor predicting patient satisfaction revealed that there is a relationship between the strategies used to reduce wait times and patient satisfaction (Agbenorku, 2013; Fournier et al., 2012; Prentice, Fincke, Miller, \& Fizer, 2011; Unger, 2011). These findings indicate that there is a need to reduce wait times for patients in many health care settings. Prentice et al. (2011) examined the relationship between hemoglobin A1C levels and the number of days that patients waited to see a primary care provider. The authors discovered that the A1C levels of participants who experienced longer wait times for primary care increased, whereas those who experienced shorter wait times had opposite results. This confirms that shorter wait times benefit patients' conditions.

\subsection{Time Flow Tools}

Some authors examined time flow and quality rating tools for care in different settings (Fournier et al., 2012; Murphy \& Evans, 2012). A quality rating tool was used to measure how patients felt about longer waits and shorter visit times with providers. Others adopted a quality improvement tool and patient flow analysis to identify inefficiencies during patient visits. The results confirmed that patient flow analysis was an effective technique to identify inefficiencies in patient visit flow. Each of these studies suggests that there is a need to examine wait times using different tools and there are interventions that warrant good results for improving access to quality care (Chen et al., 2015; Dinesh et al., 2013; Vasquez et al., 2009). For this clinical improvement project, a retrospective chart review was adopted to improve wait times and quality of care.

A Continuous Quality Improvement (CQI) framework requires a constant review of the processes involved in quality improvement in four continuous stages: Plan, Do, Check, and Act (The W. Edward Deming Institute, 2014). The Plan stage includes setting a goal and identifying the problem and steps to be taken. The Do stage includes the actions focused on improving the identified problems. The Check stage focuses on using the available data to implement actions and review whether those actions are successful. Finally, the Act stage evaluates whether steps taken meet the standards required for quality. This framework was adopted to make corrections in the clinical setting as contributory factors are identified and to answer question \#3 in the study objectives.

\section{Methods}

\subsection{Sample}

A total of 300 charts were randomly selected from the electronic health record (EHR) database at a local hospital wound care clinic in Atlanta Georgia metropolis, United States of America. These were selected from a database of patients who received treatment for wounds or ulcers between September 1, 2013, and December 31, 2013. One hundred and twenty (120) out of the 300 charts met the following inclusive criteria and were selected for retrospective review:

- Patients ages 19 to 99 years.

- patients not currently receiving treatment

- patients who had been diagnosed for treatment for one of six specified conditions

These specified conditions were wound-related diagnosis of venous insufficiency ulcer, diabetic foot ulcer, traumatic wound, arterial ulcer, surgical wound, or pressure ulcer. 


\subsection{Variables}

The variables used to guide this project were wait times, wound-related diagnosis and providers/clinicians. The variables are defined below:

\subsection{Wait Times}

The procedure for seeing patients at the wound clinic for treatment includes check-in with the clinic secretary; waiting to be seen by clinician/provider time; treatment time with clinician or provider; and check-out with the clinic secretary. The clinic specified the acceptable 'waiting to be seen by clinician/provider' time to be 15 minutes. The treatment time with provider/clinician is determined by the type of treatment provided which ranges between 45-90 minutes.

For this study, wait time is defined as unbudgeted time. To understand the unbudgeted time, the budgeted time is illustrated in Table 1.

Table 1. Budgeted times by clinic visit

\begin{tabular}{llll}
\hline Care Type & Wait Time & Treatment Time & Budgeted Time \\
\hline New Patient Evaluation & 15 minutes & 90 minutes & 105 minutes \\
Follow up visit-wound care & 15 minutes & 60 minutes & 75 minutes \\
Follow up visit-OT/PT & 15 minutes & 45 minutes & 60 minutes \\
\hline
\end{tabular}

Budgeted time is measured by clinic secretary's record of patient's check-in and check-out. The time lapse between these two signatures is measured as 'actual clinic time.'

Unbudgeted time is the difference between the budgeted time and the actual clinic time. For example if actual visit time is 120 minutes and the patient is completing a follow up visit with PT clinician, the 'wait time' or 'unbudgeted time' as defined in this study will be $120-60=60$ minutes. A checklist was developed by the PI to retrieve the above information from patients' chart.

\subsection{Wound Related Diagnosis}

Wound-related diagnosis was retrieved from the patients' charts according to provider's documented diagnosis. The six related diagnosis were venous wound; diabetic ulcer; traumatic wound; arterial ulcer; surgical wound; and pressure ulcer.

\subsection{Providers/Clinicians}

These are the providers and clinicians from this particular clinic seeing patients on a regular basis. The number of providers/clinicians are provided in the parenthesis: Physicians -MD (1); podiatrists-PD (1); nurse practitioners NP (2); wound care certified nurses-RN (2); Physical therapists- PT (3); and Occupational therapists- OT (1).

\subsection{Hypothesis}

It is hypothesized that the wait times will be explained by the type of provider/clinician and wound-related diagnosis.

\subsection{Procedure}

Approval was sought and received from the Institutional Review Board of the hospital housing the wound care clinic. As many as 300 patients' charts were randomly selected from all patients attending the wound care clinic between September 1 and December 31, 2013. The charts were reviewed for inclusion criteria. One hundred and twenty charts met the criteria and were reviewed for wait times as defined by the study, wound-related diagnosis and provider/clinicians caring for the patients during the specified time. Where the patient had multiple visits, the provider/clinician with the most visits was selected and the wait time utilized for the visit.

The data were analyzed using ANOVA to compare wait times to wound-related diagnosis and to provider/clinician. The data were analyzed using SPSS Statistics Grad Pack 22.0. Level of significance was 0.05. 


\section{Results}

Table 2. Mean wait times per wound-related diagnosis

\begin{tabular}{lccc}
\hline Diagnosis & Mean wait times & SD & N \\
\hline Venous ulcer & 27.16 & 36.76 & 25 \\
Diabetic ulcer & 17.94 & 18.34 & 17 \\
Traumatic wound & 12.45 & 36.35 & 11 \\
Arterial ulcer & 19.67 & 18.88 & 3 \\
Surgical wound & 11.69 & 27.44 & 52 \\
Pressure ulcer & 17.92 & 25.56 & 12 \\
Total & $\mathbf{1 6 . 6 9}$ & $\mathbf{2 9 . 2 2}$ & $\mathbf{1 2 0}$ \\
\hline
\end{tabular}

Table 2 highlights the unbudgeted time for wound-related diagnosis. Analysis of variance showed that wound-related diagnosis accounted for only $4 \%$ of the variance in wait times, $F(5,114)=1.009, p=0.416$. Though not significant, patients with venous ulcer have the longest wait times (27.16 \pm 36.76$)$; while traumatic wound and surgical wound have the least wait times $(12.45 \pm 36.35 \& 11.69 \pm 27.44)$

Table 3. Mean wait times per provider/clinician

\begin{tabular}{cccc}
\hline Provider/Clinician & Mean wait times & SD & $\mathrm{N}$ \\
\hline MD & 15.42 & 29.640 & 105 \\
PD & 25.60 & 25.210 & 15 \\
Total & $\mathbf{1 6 . 6 9}$ & $\mathbf{2 9 . 2 2 3}$ & $\mathbf{1 2 0}$ \\
RN & 19.43 & 31.12 & 28 \\
PT & 20.26 & 29.79 & 57 \\
OT & -5 & & 1 \\
NP & 9.08 & 25.03 & 34 \\
Total & $\mathbf{1 6 . 6 9}$ & $\mathbf{2 9 . 2 2 3}$ & $\mathbf{1 2 0}$ \\
\hline
\end{tabular}

Table 3 highlights the wait time for providers/clinicians. The relationship between wait times and providers (MD $\&$ PD) were also not significant, $F(1,118)=1.601, p=0.208$. Providers accounted for only $1 \%$ of the variance in wait times. As stated in Table 4 below, other clinicians (NP, RN, PT \& OT) accounted for $8 \%$ of the variance in wait times, $F(7,112)=1.444, p=0.195$. Though these clinicians had the highest variance, this was not a significant factor for patient wait times. The podiatrist seems to have the highest wait times $(25.60 \pm 25.21)$ while the only occupational therapist of the group finished in record time (-5).

Table 4. Summary of ANOVA Results

\begin{tabular}{lcc}
\hline Independent Variables & f & Significance Level \\
\hline Diagnoses & 1.009 & 0.416 \\
Providers & 1.601 & 0.208 \\
Clinicians & 1.444 & 0.195 \\
\hline
\end{tabular}

Note. $\mathrm{p} \leq 0.05$

\section{Discussions}

The result of this study did not support the hypothesis formulated. Neither the wound-related diagnosis or the provider/clinician contributed significantly to the variance in wait times. The standard deviation showed that there is a lot of variability with wait times irrespective of provider, clinician or diagnosis. This led the researchers to a closer look at the patient flow through the wound care clinic. To do this successfully without bias, the researchers observed the flow as the sketch in Figure 1 below:

Some of the limitations of this study are the small study sample size and the large variability within the wait times which may be a factor of how the check-in and check-out times were documented. It is possible that some patients may forget to check out on time because they are socializing with other patients or they may check out only when 
their transportation arrived. Because this is a retrospective study, it is difficult to control how and when these times were documented. A cross-sectional study measuring the wait times in real time may result in fewer variability and more reliable data.

Using the continuous quality improvement (CQI) framework of Plan, Do, Check, and Act (The Edward Deming Institute, 2014); the researchers performed the following observations to identify the problem with wait times:

- Observe arrival of patients, the actual time of electronic check-in by the clinic secretary, patient's time engagement prior to transfer into the examination room.

- Observe the actual time of the patient-provider encounter. Note any interruption on a clip board and use it to formulate guidelines on patient flow for the clinic.

- Observe the time of dismissal and the actual check out time for total encounter time and interruptions during patient-provider encounter time. Use any identified issues relating to providers to improve wait times outcome.

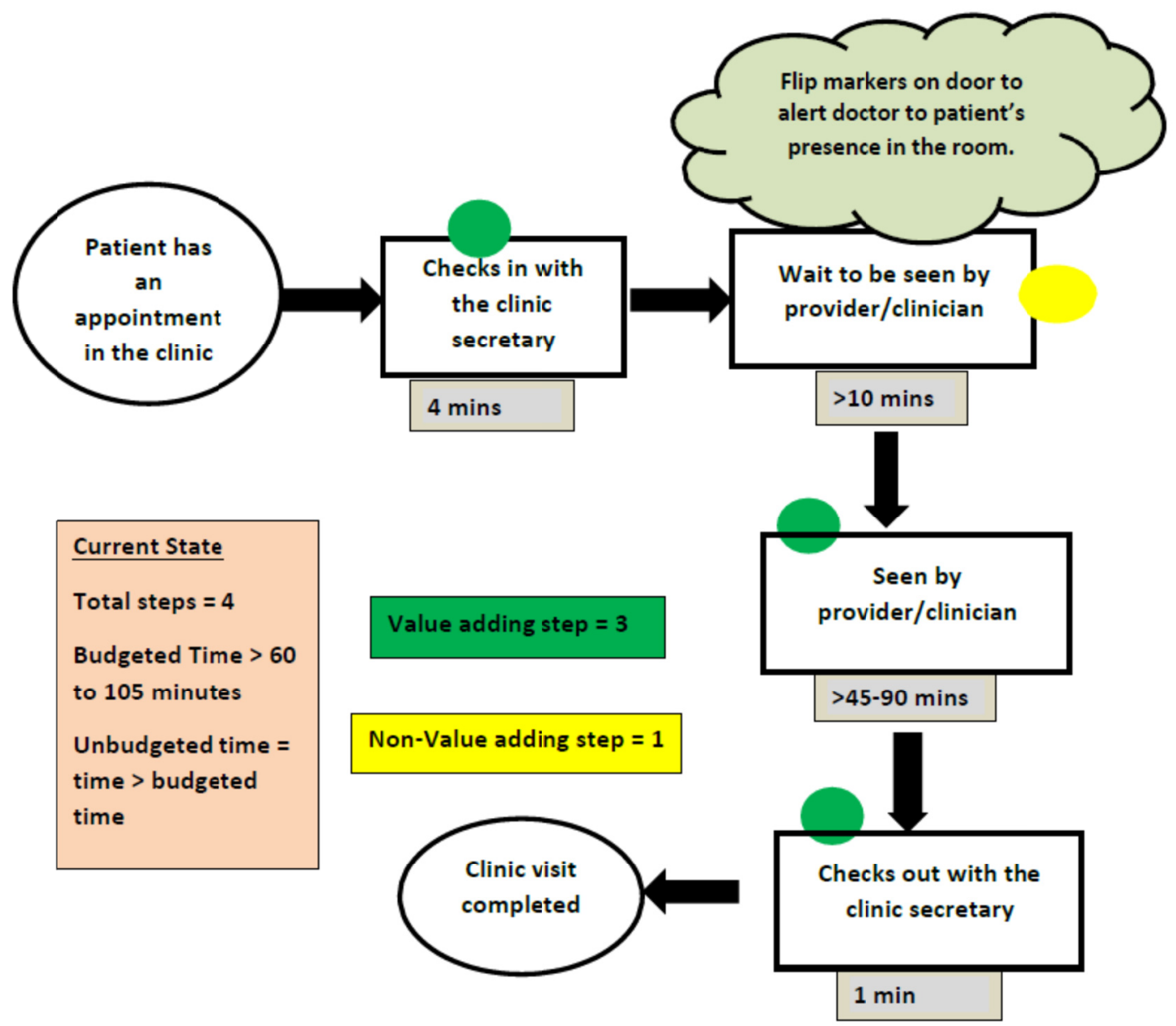

Figure 1. A priori patient flow process in wound care clinic

These observations resulted in the following identified problems and solutions:

1). Problem: The front desk personnel were slow or sometimes failed to communicate to the technician(s) about patients' check-in status and readiness for examination room.

Solution: Computer used for documentation was programmed to give electronic alert to remind secretary to inform technician(s) of patient's arrival and readiness to see provider/clinician.

2). Problem: The door flags were not flipped to indicate the examination room is empty and ready for another patient. This delayed the use of the room for other patients. 
Solution: Providers and clinicians were re-educated about ensuring that door flags are appropriately and promptly flipped.

3). Problem: Closed examination rooms indicate room in use. When exiting examination rooms, patients usually closed the door without knowledge of the need to leave the door open.

Solution: clinicians encouraged to escort their patients to check out desk and ensure that the examination room doors are left open for cleaning and next patient's use.

The patient's flow was restructured based on solutions recommended above to decrease wait time and improve patient's satisfaction (see Figure 2 below).

In summary, though there was no significant relationship between wait times, providers/clinicians, and wound-related diagnosis; this study result was a good eye-opener for the clinic to look into the patient flow process and make significant changes that improved their patients' satisfaction. Though not formally measured; after 4 weeks of implementing these changes, patients seem satisfied with the changes, and there was a noticeable decrease in wait times. One patient commented "I have never been to a clinic like this where I get to see a provider within the scheduled time and I can see a few doctors on the same day.

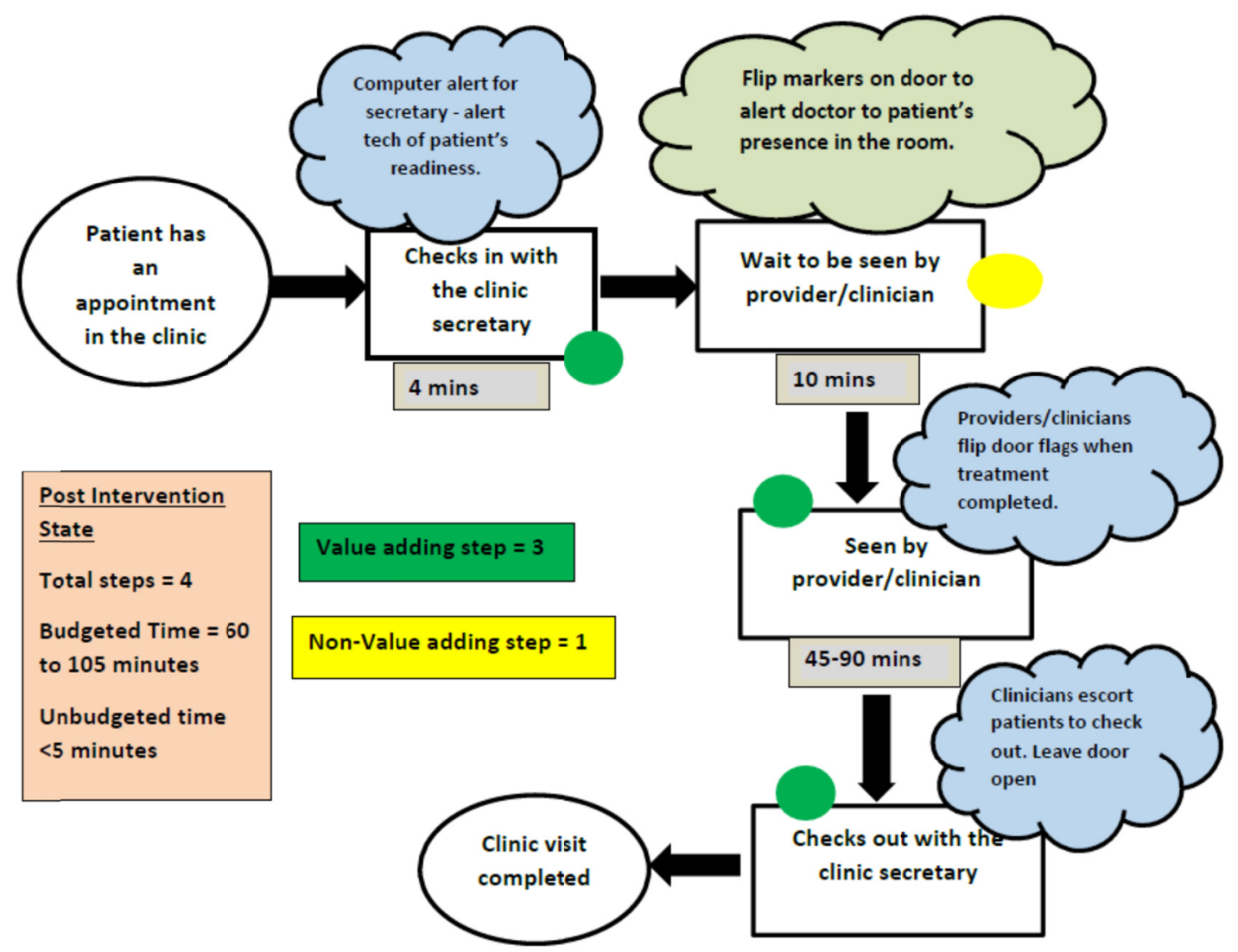

Figure 2. Post-intervention patient flow process in wound care clinic

\section{Conclusions}

In conclusion, improving wait times has the potential to improve the quality of care and increase timely access to that care. Analysis of our data reveals that there is a weak connection between the wait time, the patient diagnoses, the clinicians, and the providers. Although these connections are weak, other areas have been found to affect patient flow times, including delays in relaying communication and interdepartmental transfer of critically ill patients.

\section{Clinical Recommendations}

Quality improvement initiatives are diverse; therefore, it is important to support quality improvement strategies, 
however small they may be. For this project, it was possible to prioritize and improve communication by creating a chart for effective patient flow through the clinic. This also allows the designation of a clinician on a daily basis to supervise the interdepartmental transfer of critically ill patients to free other staff members to care for patients and to reduce the likelihood for longer wait times.

\section{References}

Agbenorku, P. (2013). Quality assurance in wound management in a developing country: How satisfied are our patients? Surgical Science, 4, 39-44. https://doi.org/10.4236/ss.2013.41007

Bleustein, C., Rothschild, D.B., Valen, A., Valaitis, E., Schweitzer, L., \& Jones, R. (2014). Wait times, patient satisfaction scores, and the perception of care. American Journal of Managed Care, 20(5), 393-400. Retrieved from https://www.ncbi.nlm.nih.gov/pubmed/25181568

Chen, Y.T, Chang, C.C., Shen, J.H., Lin, W., \& Chen, M.Y. (2015). Demonstrating a conceptual framework to provide efficient wound management service for a wound care center in a tertiary hospital. Medicine, 94(44), e1962. https://doi.org/10.1097/MD.0000000000001962

Dinesh, T.A., Singh, S., Nair, P., \& Remya, T.R. (2013). Reducing waiting time in outpatient services of large university teaching hospital-A six sigma approach. Management in Health, 17(1), 31-37. Retrieved from http://journal.managementinhealth.com/index.php/rms/article/viewFile/259/822

Esbenshade, A. (2015). Making the middle count: Three tools to improve throughput for a better patient $\begin{array}{lllll}\text { experience. } \quad \text { Advanced } & \text { Emergency }\end{array}$ https://doi.org/10.1097/TME.0000000000000049

Fournier, J., Heale, R., \& Rietze, L. (2012). I can't wait: Advanced access decreases wait times in primary healthcare. Healthcare Quarterly, 15(1), 64-68. https://doi.org/10.12927/hcq.2012.22763

McHugh, M., Van Dyke, K., McClelland, M., \& Moss, D. (2011, October). Improving patient flow and reducing emergency department crowding: A guide for hospitals. Rockville, MD: Agency for Healthcare Research and Quality. Retrieved from http://www.ahrq. gov/research/findings/final-reports/ptflow/index.html

Michael, M., Schaffer, S.D., Egan, P.L., Little, B.B., \& Pritchard, P.S. (2013). Improving wait times and patient satisfaction in primary care. Journal for Healthcare Quality, 35(2), 50-59. https://doi.org/10.1111/jhq.12004

Murphy, P.S., \& Evans, G.R. (2012). Advances in wound healing: A review of current would healing products. Plastic Surgery International. https://doi.org/10.1155/2012/190436

Oredsson, S., Jonsson, H., Rognes, J., Lind, L., Goransson, K.E., Ehrenberg, A., ... Farrohknia, N. (2011). A systematic review of triage-related interventions to improve patient flow in emergency departments. Scandinavian Journal of Trauma, Resuscitation and Emergency Medicine, 19, 43. https://doi.org/10.1186/1757-7241-19-43

Patient Protection and Affordable Care Act, 42 U.S.C. $\S 18001$ (2010). Retrieved from https://www.law.cornell.edu/wex/patient_protection_and_affordable_care_act_of_2010

Prentice, J.C., Fincke, B.G., Miller, D.R., \& Pizer, S.D. (2011). Outpatient wait time and diabetes care quality improvement. American Journal of Managed Care, 17(2), e43-e54. Retrieved from https://www.ncbi.nlm.nih.gov/pubmed/21473659

The W. Edward Deming Institute. (2014). The PDCA Cycle. Retrieved from https://www.deming.org/theman/theories/pdsacycle

Unger, P.G. (2011). Improving patient satisfaction in the clinic setting. Today's Wound Clinic, 5(9). Retrieved from http://www.todayswoundclinic.com/articles/improving-patient-satisfaction-clinic-setting

Vasquez, S.R., Campbell, J., Haman, G., George, C., \& Sprabery, L. (2009). Anticoagulation clinic workflow

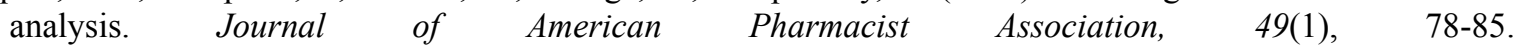
https://doi.org/10.1331/JAPhA.2009.07086

\section{Copyrights}

Copyright for this article is retained by the author(s), with first publication rights granted to the journal.

This is an open-access article distributed under the terms and conditions of the Creative Commons Attribution license (http://creativecommons.org/licenses/by/4.0/). 